\title{
Solitary pulmonary metastasis of pancreatic cancer presenting a thin-walled cavity
}

\author{
Katsunari Matsuoka, Mitsuhiro Ueda, Yoshihiro Miyamoto \\ Department of Thoracic Surgery, National Hospital Organization Himeji Medical Center, Himeji, Japan
}

Received April 01, 2015; Revised April 12, 2015; Accepted April 22, 2015; Published Online June 10, 2015

\section{Case Report}

\begin{abstract}
Because the prognosis of the patients with pancreatic cancer is very poor, there are few opportunities to perform surgery for pulmonary metastasis. Here we report the resection of a solitary pulmonary metastasis from pancreatic cancer, appearing as thin-walled cavity, which appeared 5 years after pancreaticoduodenectomy. Although the present patient underwent incomplete resection because of malignant pleural effusion and pleural dissemination, he is currently still alive without evident recurrence at 18 months after surgery.
\end{abstract}

Keywords: Pancreatic Cancer; Pulmonary; Metastasis; Cavity; Solitary

\section{Introduction}

The prognosis of patients with advanced pancreatic cancer is still poor and there are few opportunities to perform surgery for pulmonary metastasis of pancreatic cancer. ${ }^{1,2,3}$ We have experienced a rare case of solitary pulmonary metastasis presenting as a thin-walled cavity, which emerged 5 years after initial surgery and the patient is currently alive without evident recurrence at one year and 6 months after surgery instead of malignant pleural effusion and pleural dissemination.

\section{Case presentation}

A 70-year-old male was referred to our hospital because of a pulmonary thin-walled cavity lesion. He had undergone pancreaticoduodenectomy for pancreatic cancer 5 years previously, when histological examination had revealed stage $4 \mathrm{~b}$ disease with paraaortic lymph node metastasis. Despite adjuvant chemotherapy with gemcitabine for 1 years after surgery, the level of CA19-9 had increased and liver metastasis had appeared. TS-1 was then administered for 2 years, and this led to disappearance of the liver metastasis and decrease of CA19-9 to a normal level. For 2 years thereafter, the patient had been followed up by routine chest and abdominal CT without any treatment due to severe TS-1 related dermatitis. At 2 years after withdrawal of TS-1, a thin walled cavity lesion had appeared in the lower lobe of the right lung, and this had gradually increased in size. He was then referred to our hospital for further examination.
Chest CT on admission demonstrated a thin-walled cavity lesion about $27 \mathrm{~mm}$ in diameter in the right lower lobe. (Figure 1A, 1B) The patient was a smoker, with a pack-year index of 90. The levels of tumor markers (carcinoembryonic antigen, Cyfra and CA19-9) on admission were within normal limits. Routine blood counts and other biochemical parameters were also within normal limits. Transbronchial biopsy under bronchofiberscope guidance was unable to provide a clear diagnosis. Because the possibility of primary lung cancer could not be excluded, thoracoscopic surgery was performed. This revealed mild serous pleural effusion and multiple small white nodules on the visceral and parietal pleura. (Figure 1C, 1D) Cytological and histological examination showed malignant cells in pleural effusion and small white nodules. We performed video-assisted thoracoscopic wedge resection of the lung and intrathoracic administration of cisplatin. Histological examination of the resected lung mass demonstrated proliferation of large, clear columnar epithelial cells with nuclear atypia arranged in atypical glandular structures. Immunohistochemical staining of CK7 and CA19-9 was positive, CK20 was slightly positive, and TTF-1 and NapsinA was negative. (Figure 2) On this basis, the tumor was diagnosed as metastatic adenocarcinoma from pancreatic cancer. The patient was discharged from hospital 3 days after surgery without any postoperative complications. After 2 months of TS-1 administration following surgery, he has been followed up at local hospital. One year and 6 month after surgery, he still alive without evident recurrence. 


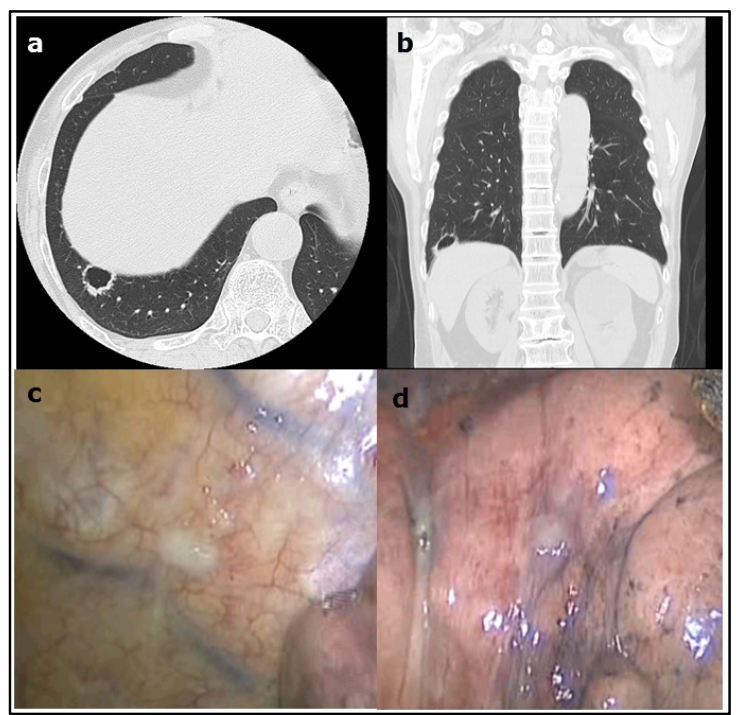

FIG. 1: Chest computed tomography on admission demonstrated a thin-walled cavity lesion about $27 \mathrm{~mm}$ in diameter in the right lower lobe with peripheral tissue indentation. (a: axial view, b: coronal view) Intraoperative video-assisted thoracoscopic image demonstrated dissemination of metastatic pancreatic cancer. (c: dissemination at parietal pleura, d: dissemination at visceral pleura).

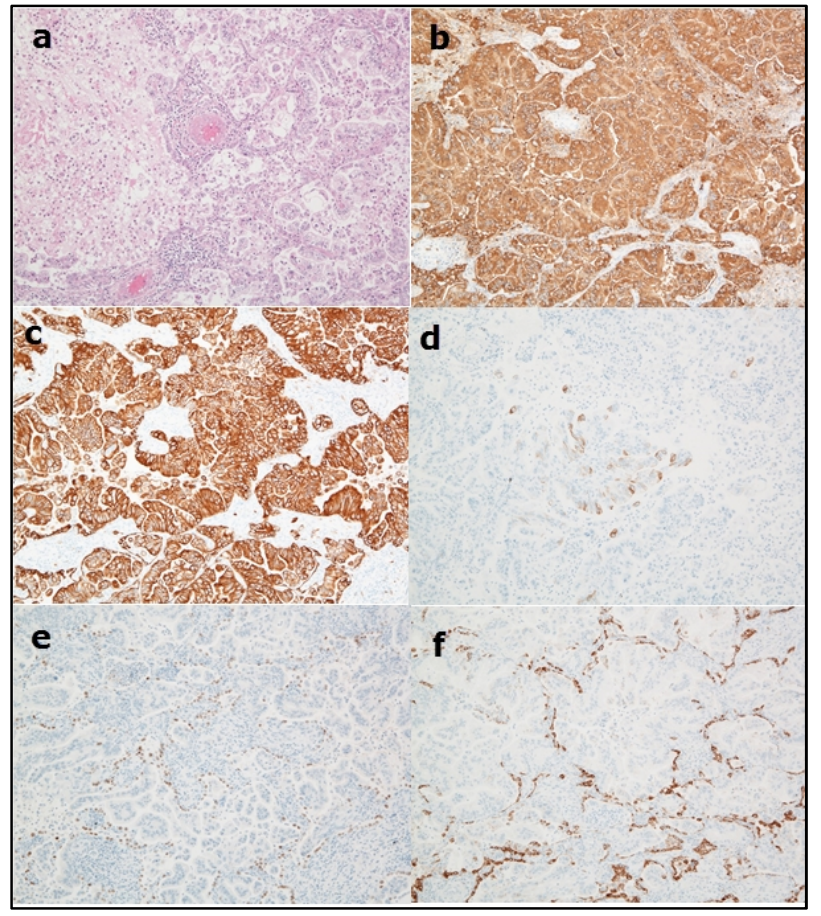

FIG. 2: Histological examination of the resected lung tumor demonstrated proliferation of large, clear columnar epithelial cells with nuclear atypia arranged in atypical glandular structures (a: H-E stain $\times 100$ ). Immunohistochemical staining of CK7 (b) and CA19-9 (c) was positive, CK20 (d) was slightly positive, and TTF-1 (e) and Nap$\sin \mathrm{A}(\mathrm{f})$ was negative.

\section{Discussion}

It has been reported that $92 \%$ of cavity lesion with a wall thickness of less than $4 \mathrm{~mm}$ are benign disorders. ${ }^{4}$ Although primary lung cancer often shows cavity formation within the tumor, this feature is rare in cases of pulmonary metastasis from cancers in other organs. Most reported metastatic pulmonary lesions showing cavities have originated form head and neck sarcoma or colon cancer. ${ }^{5}$ Solitary pulmonary metastasis of pancreatic cancer presenting a thin-walled cavity was not reported previously. In this present case, CT findings suggested a primary lung carcinoma or infectious disease, as the lesion was a solitary cavity with a wall less than $4 \mathrm{~mm}$ thick and slight peripheral tissue indentation.

The prognosis of advanced pancreatic cancer is still poor, and the median survival for patients after pancreatic resection is 12.6 months. Among patients who undergo surgical resection, the majority die due to disease recurrence, with a 3 -year disease-free survival rate of only $27 \% .{ }^{1}$ Because venous blood from the pancreas flowed into portal system, distant metastasis of pancreatic cancer usually appeared in liver and peritoneal cavity, and lung metastasis is rare, with a reported incidence of $6.4 \%{ }^{6}$ Moreover, most patients with lung metastasis from pancreatic cancer are not considered for surgical treatment because of coexisting involvement of other organs or multiple lung tumors. Therefore, resection of lung metastasis from pancreatic cancer is performed very rarely, and reported in only a few cases. ${ }^{1,2}$ In this present case, because liver metastasis had completely regressed after chemotherapy, no form of organ involvement other than that of the lung was evident on admission, and preoperative CT findings suggested a primary lung carcinoma or infectious disease, the patient underwent surgical treatment.

Some reports have documented long-term survival after complete resection of pulmonary metastasis from pancreatic cancer. ${ }^{1}$ Although the present patient underwent incomplete resection because of malignant pleural effusion and pleural dissemination, he is currently still alive without evident recurrence at 18 months after surgery. In this case, multimodality treatment including resection of solitary pulmonary nodule, intrathoracic administration of anti-cancer drug and postoperative chemotherapy may be effective for the treatment of lung metastasis of pancreatic cancer with malignant pleural effusion and dissemination. If patients with pancreatic cancer have solitary pulmonary metastasis without involvement of other organs, surgical resection may be feasible.

Although intraoperative histological examination did not provide a precise diagnosis, postoperative immunohistochemical staining demonstrated that the lesion was a metastasis from pancreatic cancer. Immunohistochemical staining of CK7 and CA19-9, which are usually positive in pancreatic cancer, gave a positive result, and CK20 was also slightly positive, whereas TTF-1 and NapsinA, which are usually positive in lung cancer, were negative. Immunohistochemical staining was useful to distinguishing between lung metastasis of pancreatic cancer and primary lung carcinoma. 


\section{Conclusion}

In conclusion, we have described a rare case of solitary pulmonary metastasis with a thin-walled cavity arising 5 years after pancreaticoduodenectomy for pancreatic cancer.

\section{Conflict of interest}

The authors declare that they have no conflicts of interest. The authors alone are responsible for the content and writing of the paper.

\section{References}

1. Arnaoutakis GJ, Rangachari D, Laheru DA, et al. Pulmonary resection for isolated pancreatic adenocarcinoma metastasis: an analysis of outcomes and survival. J Gastrointest Surg 2011;15:1611-7.

2. Kitasato Y, Nakayama M, Akasu G, et al. Metastatic pulmonary adenocarcinoma 13 years after curative resection for pancreatic cancer: report of a case and review of Japanese literature. JOP 2012;13:296-300.
3. Giakoustidis A, Cherian PT, Zen Y, et al. Metastatic pulmonary adenocarcinoma 6 years after curative resection for ampullary adenocarcinoma. Metastatic disease from initial primary or metachronous tumour? JOP 2011;12:32-6.

4. Woodring JH, Fried AM, Chuang VP. Solitary cavities of the lung; diagnostic implications of cavity wall thickness. AJR Am Roentogenol 1980;135:1269-71.

5. Dodd GD, Boyle JJ. Excavating pulmonary metastases. Am J Roentgenol Ther Nucl Med 1961;85:277-93.

6. Sperti C, Pasquali C, Piccoli A, Pedrazzoli S. Recurrence after resection for ductal adenocarcinoma of the pancreas. World J Surg 1997;21:195-200. 